\title{
Demonstration of a functional variant of chloramphenicol acetyltransferase in Haemophilus influenzae
}

\author{
M. D. SMITH and M. C. KELSEY
}

Department of Microbiology, Whittington Hospital, Highgate Hill, London N19 5NF

\begin{abstract}
Summary. Seven clinical isolates of chloramphenicol-resistant Haemophilus influenzae were studied. The products of chloramphenicol inactivation by chloramphenicol acetyltransferase (CAT) were identified by high performance liquid chromatography. The sole product in $H$. influenzae is a single monoacetyl compound, whereas variants of CAT isolated from other chloramphenicol-resistant bacteria usually produce both monoacetyl and diacetyl chloramphenicol metabolites. The chloramphenicol resistance gene was found to reside on a $65-\mathrm{kb}$ plasmid which, in five of the six cases studied, appeared to be integrated into the host cell chromosome.
\end{abstract}

\section{Introduction}

Haemophilus influenzae is a cause of acute meningitis and epiglottitis in young children. The high incidence of ampicillin resistance in this organism has led to the continued use of chloramphenicol as a treatment of first choice. Chloramphenicol-resistant strains of $H$. influenzae are unusual but well recognised (Margués et al., 1984; Powell et al., 1987). Conjugative transfer of chloramphenicol resistance to both Escherichia coli and $H$. influenzae recipient strains is well established (van Klingeren et al., 1977; Roberts et al., 1980), although studies have demonstrated that plasmids are not consistently detected in the donor strains (Shaw et al., 1978; Stuy, 1979).

Resistance to chloramphenicol in $H$. influenzae may be due to a permeability barrier, as suggested by Burns et al. (1985), but is more frequently the result of acetylation by chloramphenicol acetyltransferase (CAT) (Roberts et al., 1980). Studies of E. coli (Shaw, 1967), Staphylococcus aureus (Shaw and Brodsky, 1968) and Streptococcus faecalis (Nakagawa et al., 1979) have demonstrated that CAT variants catalyse the acetyl coenzyme A (CoA)-dependent acetylation of chloramphenicol resulting in the initial major product, 3-acetyl chloramphenicol, which rearranges non-enzymically to yield 1-acetyl chloramphenicol, a substrate for the second acetylation to yield 1,3-diacetyl chloramphenicol (Shaw, 1983).

Received 3 Nov. 1987; revised version received 15 Aug. 1988; accepted 16 Feb. 1989.
The activity and metabolic products of the enzyme derived from $H$. influenzae are poorly documented. The present study was designed to characterise further some of the properties of CAT derived from $H$. influenzae.

\section{Materials and methods}

\section{Bacterial strains}

The bacterial strains used in this study are listed in the table. Only $H$. influenzae strain 2035 was Pitman capsular type b. $H$. influenzae strain 1388 is a plasmid recipient strain $(\operatorname{Rec} A)$ which does not allow the integration of any acquired plasmid into the chromosome. $E$. coli $\mathrm{K} 12$ strain 525 is a lactose-fermenting plasmid recipient and $E$. coli strain W677/R5 (NCTC 11186) is a known type-I CAT producer (Shaw W V, personal communication). Although CAT from $H$. influenzae has similarities to typeII CAT (Shaw, 1983), a suitable type-II CAT control was not available for inclusion in this study.

\section{Media}

Disk sensitivity tests and minimum inhibitory concentrations (MICs) for $E$. coli were performed on Diagnostic Sensitivity Test (DST) Agar (Oxoid). Sensitivity tests and MICs for $H$. influenzae were performed on DST agar containing "chocolated" horse blood $10 \%$. Fluid cultures were in Digest Broth (Southern Group Laboratories), with the addition of IsoVitaleX (BBL Microbiology Systems) $3 \% \mathrm{v} / \mathrm{v}$ for $H$. influenzae. Chloramphenicolresistant transconjugant strains were cultured on selective media containing the appropriate antibiotics. 
Table . Chloramphenicol acetyltransferase (CAT) activity and susceptibilities of clinical isolates of chloramphenicolresistant $H$. influenzae, and their $E$. coli and $H$. influenzae transconjugants

\begin{tabular}{|c|c|c|c|c|c|c|c|}
\hline \multirow[b]{2}{*}{ Strain } & \multirow[b]{2}{*}{$\begin{array}{c}\text { Resistance } \\
\text { pattern }\end{array}$} & \multicolumn{2}{|c|}{ Original isolate } & \multicolumn{2}{|c|}{$\begin{array}{c}\text { H. influenzae } \\
\text { transconjugants }\end{array}$} & \multicolumn{2}{|c|}{ E. coli transconjugants } \\
\hline & & $\begin{array}{c}\text { CAT activity } \\
(\mu \mathrm{mol} / \mathrm{min} / \\
\left.10^{9} \text { orgs }\right)\end{array}$ & $\begin{array}{c}\text { Chloram- } \\
\text { phenicol } \\
\text { MIC }(\mathrm{mg} / \mathrm{L})\end{array}$ & $\begin{array}{c}\text { CAT activity } \\
\text { ( } \mu \mathrm{mol} / \mathrm{min} / \\
\left.10^{9} \mathrm{orgs}\right)\end{array}$ & $\begin{array}{l}\text { Chloram- } \\
\text { phenicol } \\
\text { MIC }(\mathrm{mg} / \mathrm{L})\end{array}$ & $\begin{array}{c}\text { CAT activity } \\
(\mu \mathrm{mol} / \mathrm{min} / \\
\left.10^{9} \text { orgs }\right)\end{array}$ & $\begin{array}{l}\text { Chloram- } \\
\text { phenicol } \\
\text { MIC }(\mathrm{mg} / \mathrm{L})\end{array}$ \\
\hline \multicolumn{8}{|l|}{ H. influenzae } \\
\hline 2035 & Cm Tc Amp & $2 \cdot 56$ & 4 & - & - & - & - \\
\hline W1 & $\mathrm{Cm} \mathrm{Tc}$ & 0.47 & 8 & 3.09 & 16 & 1.64 & $>256$ \\
\hline W2 & $\mathrm{Cm} \mathrm{Tc}$ & 0.70 & 16 & 1.52 & 16 & $5 \cdot 76$ & $>256$ \\
\hline W3 & $\mathrm{Cm} \mathrm{Tc}$ & 0.71 & 8 & $1 \cdot 40$ & 16 & $3 \cdot 36$ & $>256$ \\
\hline W4 & $\mathrm{Cm} \mathrm{Tc}$ & $1 \cdot 31$ & 4 & $8 \cdot 27$ & 16 & 1.95 & $>256$ \\
\hline RN5 & $\mathrm{Cm} \mathrm{Tc}$ & 0.38 & 8 & 2.74 & 16 & 2.75 & 64 \\
\hline A6 & $\mathrm{Cm}$ Tc Amp & 0.60 & 16 & 0.60 & 16 & - & - \\
\hline \multicolumn{8}{|l|}{ Recipients } \\
\hline H. influenzae 1388 & Str & 0 & 0.5 & $\ldots$ & $\ldots$ & $\ldots$ & $\ldots$ \\
\hline E. coli $\mathrm{K} 12525$ & $\mathrm{Nal}$ & 0 & 2 & $\ldots$ & $\ldots$ & $\ldots$ & $\ldots$ \\
\hline \multicolumn{8}{|l|}{ Control } \\
\hline E. coli $\mathrm{W} 677 / \mathrm{R} 5$ & $\mathrm{Cm} \mathrm{Str}$ & 0.93 & $>256$ & $\ldots$ & $\ldots$ & $\ldots$ & . \\
\hline
\end{tabular}

$\mathrm{Cm}$, chloramphenicol; Tc, tetracycline; Amp, ampicillin; Str, streptomycin; Nal, nalidixic acid.

$-=$ No transfer obtained $; \ldots=$ not tested.

\section{Determination of MICs}

MICs for all donor, recipient and transconjugant strains were determined by an agar dilution method. The strains were applied with a multipoint inoculator at inocula of $10^{5} \mathrm{cfu} / \mathrm{spot}$. The MICs of chloramphenicol (Parke-Davis), tetracycline (Lederle) and ampicillin (Beecham) were determined.

\section{Transfer of drug resistance}

Each donor strain of $H$. influenzae was mated with the $E$. coli recipient strain 525 and also with the $H$. influenzae recipient strain 1388 . Filter matings were performed by a modification of the method of van Klingeren et al. (1977). Donor and recipient strains were grown overnight $(18 \mathrm{~h})$ in the appropriate broth. Samples $(1 \mathrm{ml})$ of a $1: 1$ mixture of donor and recipient cultures were filtered through cellulose filters $(25 \mathrm{~mm}$ diameter, $0.22 \mu \mathrm{m}$ pore size). The filter was removed and incubated for $18 \mathrm{~h}$ on chocolated blood agar in $\mathrm{CO}_{2} 5 \%$. The resulting growth was rinsed from the filters by agitation in $1 \mathrm{ml}$ of digest broth and samples of the suspension were plated on to the appropriate selective media. MacConkey Agar (Oxoid) containing chloramphenicol $20 \mathrm{mg} / \mathrm{L}$ and nalidixic acid $50 \mathrm{mg} / \mathrm{L}$ was used for the $E$. coli transconjugants and chocolate DST agar containing chloramphenicol $2 \mathrm{mg} / \mathrm{L}$ and streptomycin $20 \mathrm{mg} / \mathrm{L}$ for the $H$. influenzae transconjugants. Single colonies of the resultant transconjugants were re-cultured on selective media for confirmation before MICs were determined.

\section{Preparation and characterisation of plasmid DNA}

The isolation and detection of plasmids was performed by agarose gel electrophoresis, by the method of Kado and Liu (1981).

\section{Preparation of cell-free extracts}

Overnight $(18 \mathrm{~h})$ broth cultures were prepared $(20 \mathrm{ml})$ and viable counts were performed before harvesting the cells by centrifugation $(6000 \mathrm{~g}$ for $30 \mathrm{~min})$. The cell pellet was resuspended in $2 \mathrm{ml}$ of $0 \cdot 1 \mathrm{M}$ Tris- $\mathrm{HCl}$ buffer $(\mathrm{pH} 7 \cdot 8)$. This step was repeated and then the cells were disrupted by ultrasonication. The clear cell-free supernate obtained after high speed centrifugation $(38000 \mathrm{~g}$ for $60 \mathrm{~min}$ at $4^{\circ} \mathrm{C}$ ) was used for the assay of CAT activity.

\section{Assay of CAT activity and characterisation of metabolites}

Chloramphenicol acetyltransferase activity was assayed by the technique of Lovering et al. (1986), in which high performance liquid chromatography (HPLC) is used to detect chloramphenicol and its metabolites. Single estimations were performed for each enzyme extract.

The enzyme extracts were incubated with chloramphenicol in the presence of acetyl CoA generated by a mixture of coenzyme A, acetyl phosphate and phosphotransacetylase as described by Breeze and Simpson (1982). The final reaction mixture contained Tris- $\mathrm{HCl}$ buffer (pH 7.8)(Sigma) $100 \mu \mathrm{mol}, \mathrm{MgCl}_{2}$ (BDH) $15 \mu \mathrm{mol}$, chloramphenicol (Parke-Davis) $800 \mathrm{nmol}$, coenzyme A 
(Sigma) $0.25 \mathrm{mg}$, acetyl phosphate (Sigma) $5 \mathrm{mg}$, and phosphotransacetylase (Sigma) 25 units in a volume of $800 \mu \mathrm{l}$, to which was added $100 \mu \mathrm{l}$ of the crude enzyme extract, giving a final volume of $900 \mu$ l. The reaction mixture was incubated at $37^{\circ} \mathrm{C}$ and at timed intervals 50$\mu \mathrm{l}$ samples were removed and the acetylation reaction was stopped by the addition of an equal volume of acetonitrile (Rathburn Chemicals). These samples were then injected into the chromatograph within $8 \mathrm{~h}$ of sampling. CAT activity remains stable under these conditions (Shaw W V, personal communication). The chromatography was performed with a PU 4010 pump (Pye Unicam), a Partisil 10 ODS-3 $\mathrm{C}_{18}$ column (Whatman Ltd) and a PU 4020 UV-absorbance detector (Pye Unicam) set at $254 \mathrm{~nm}$. The mobile phase consisted of methanol : water: phosphoric acid $(20: 79: 1)$ at a flow rate of $3 \mathrm{ml} / \mathrm{min}$.

\section{Results}

\section{Resistance phenotypes and their transfer}

During the filter-mating procedures, chloramphenicol resistance was transferred from donor to both $H$. influenzae and $E$. coli recipient strains in nearly all cases. The exceptions were $H$. influenzae strain 2035 which failed to transfer resistance to either recipient strain and $H$. influenzae strain A6 which only transferred resistance to the $H$. influenzae recipient. The MICs of chloramphenicol for donor, recipient and transconjugant strains are given in the table.

Tetracycline and ampicillin resistance did not always transfer along with chloramphenicol resistance. Donor strain $H$. influenzae A6 transferred chloramphenicol, tetracycline and ampicillin resistance to the $H$. influenzae 1388 recipient but no resistance was transferred to the $E$. coli 525 recipient. Tetracycline resistance transferred with chloramphenicol resistance to the $H$. influenzae 1388 recipient in 4 of 6 cases and to the $E$. coli 525 recipient in only 2 of 5 cases.

Spontaneous resistance could not be produced in the recipient strains by serial subculture on media containing subinhibitory levels of chloramphenicol.

\section{Demonstration of plasmids}

Only one of the donor $H$. influenzae strains (W4) had a demonstrable plasmid $(65 \mathrm{~kb})$. However, all six $H$. influenzae 1388 transconjugants had plasmids of a similar size $(65 \mathrm{~kb})$, which were not present in the original $H$. influenzae 1388 recipient strain. No plasmid could be demonstrated in any of the $E$. coli 525 transconjugant strains.

\section{Characterisation of $C A T$}

Standard curves were constructed for known concentrations of chloramphenicol and its metabolite, 1,3-diacetyl chloramphenicol. In the absence of standard material of 3-acetyl chloramphenicol, the concentration was calculated on the assumption of equimolar conversion from chloramphenicol. Graphs were constructed for each enzyme extract plotting concentrations of chloramphenicol and metabolites against time. The activity of CAT in the extracts was then calculated from the initial linear section of the reaction plots. The results are shown in the table.

In reactions performed with a commercial CAT preparation (type I, derived from $E$. coli; Sigma) or an extract of $E$. coli W677/R5, a known type-I CAT producer, both the monoacetyl and diacetyl metabolites were readily demonstrated (fig. 1).

None of the extracts from the original $H$. influenzae isolates produced 1,3-diacetyl chloramphenicol under these conditions ( $\mathrm{pH} 7 \cdot 8$ ). All had demonstrable CAT but the level of activity varied between strains. In all of these cases, the only product was the monoacetyl metabolite. Varying the $\mathrm{pH}$ of the reaction mixture $(\mathrm{pH} 7 \cdot 8,8.2,8 \cdot 6$, $9 \cdot 2$ ) and lengthening the reaction time to $10 \mathrm{~h}$ did not alter the production of metabolites. Similarly, extracts from all $H$. influenzae 1388 and $E$. coli 525 transconjugants demonstrated CAT activity but failed to produce 1,3-diacetyl chloramphenicol.

Extracts of all donor and transconjugant strains were left to react for $5 \mathrm{~h}$ to ensure complete conversion of chloramphenicol to the monoacetyl metabolite, with no demonstrable 1,3-diacetyl product; 30 units of the commercial type-I CAT (Sigma) were then added to $300 \mu$ of the remaining

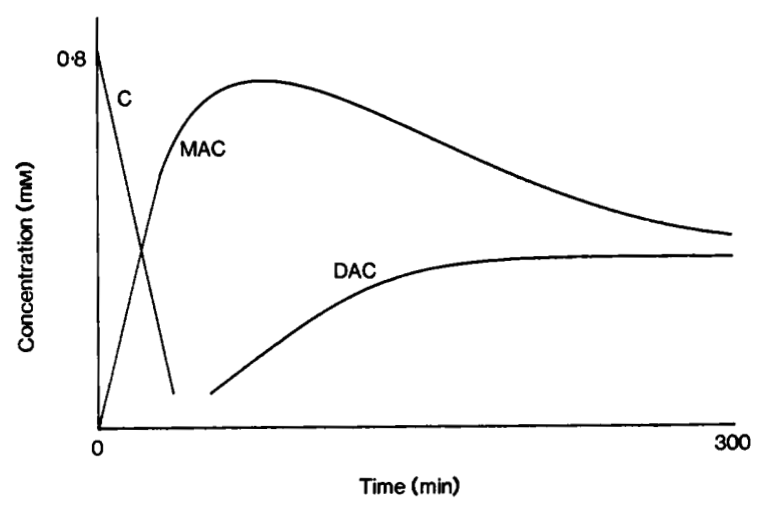

Fig. 1. The concentration/time relationship of chloramphenicol (C) and its metabolites, monoacetyl chloramphenicol (MAC) and diacetyl chloramphenicol (DAC), in a reaction mixture containing chloramphenicol, acetyl coenzyme A and type-I CAT buffered at $\mathrm{pH} 7 \cdot 8$. 


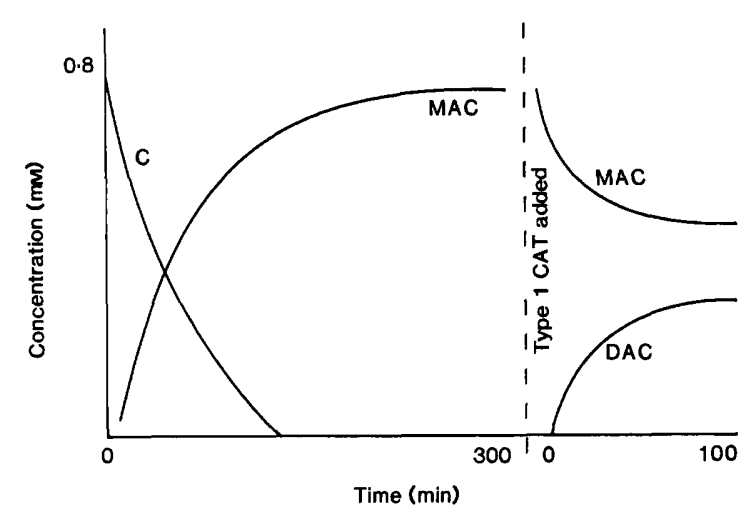

Fig. 2. The concentration/time relationship of chloramphenicol (C) and its metabolites, monoacetyl chloramphenicol (MAC) and diacetyl chloramphenicol (DAC), in a reaction mixture containing chloramphenicol, acetyl coenzyme $A$ and an extract of chloramphenicol-resistant $H$. influenzae (W1), buffered at $\mathrm{pH} 7 \cdot 8$. After $300 \mathrm{~min}$, type-I CAT was added to the reaction.

mixture and 50- $\mu$ l samples were taken at further intervals. In every case 1,3-diacetyl chloramphenicol was produced from the monoacetyl precursor (fig. 2).

\section{Discussion}

We have confirmed that chloramphenicol resistance may be transferred in vitro, presumably by conjugation, from $H$. influenzae into other strains of the same species and also into $E$. coli. All further studies were made on single transconjugants picked after mating. We were unable to demonstrate spontaneous resistance to chloramphenicol in the recipient $H$. influenzae or $E$. coli strains, but it is difficult to be sure that the strains studied were really transconjugants and not spontaneous mutants.

Although plasmid DNA could be detected in only one of the original donor strains of $H$. influenzae, it was evident in each of the $H$. influenzae 1388 transconjugants. Other workers (Shaw et al., 1978) have experienced similar difficulties and the use of different methods for plasmid extraction may have demonstrated plasmids in more of the donor strains. However the results are in agreement with the observations made by Stuy (1980), who concluded that most clinical strains of antibioticresistant $H$. influenzae carry a conjugative R plasmid which is normally integrated in the chromosome, but which may be excised to yield a few cells in each population harbouring a free plasmid. Such cells may have been responsible for the transfer of resistance observed in the mating procedure.
Chloramphenicol resistance in our clinical isolates of $H$. influenzae was observed, in each case, to be associated with the presence of CAT activity. However, there was no correlation between the level of resistance to chloramphenicol and the measured CAT activity in cell extracts. The transfer experiments also demonstrated the presence of CAT in $H$. influenzae and $E$. coli transconjugants. Levels of CAT activity were significantly higher in the $E$. coli transconjugants (Student's $t$ test, $\mathrm{p}<0.05$ ), an observation probably due to a greater number of gene copies per cell in the transconjugants. Levels of CAT activity were slightly higher in the $H$. influenzae transconjugants than in the donor strains, a result which may be due either to an increased gene copy number, or to enhanced expression when the resistance gene is transcribed from a plasmid locus rather than in the integrated (chromosomal) state (Iyobe et al., 1974). The higher CAT activity in cell extracts was associated with greatly enhanced resistance to chloramphenicol for the $E$. coli transconjugants but no such correlation was observed for the $H$. influenzae transconjugants. However, these results are based on single estimates of CAT activity so the apparent lack of correlation between MIC and CAT activity, for individual organisms, may be incorrect.

Early studies of CAT variants from different organisms have shown the production of both monoacetyl and diacetyl chloramphenicol metabolites (Shaw, 1967; Shaw and Brodsky, 1968, Nakagawa, 1981). It has been suggested that chloramphenicol is initially acetylated at the C-3 hydroxyl group to give 3-acetyl chloramphenicol, a fraction of which then undergoes spontaneous nonenzymic rearrangement at an alkaline $\mathrm{pH}$ to yield 1-acetyl chloramphenicol. This is thought to be analogous to the spontaneous rearrangement of chloramphenicol-3-monosuccinate (Brent et al., 1980); the equilibrium favours a ratio of 3-acetyl to 1-acetyl derivatives of 3:1 (Kleanthous and Shaw, 1984). A second acetylation at the C-3 hydroxyl group then yields 1,3-diacetyl chloramphenicol. This is summarised as follows:
(1) chloramphenicol + acetyl CoA CAT
3-acetyl chloramphenicol
$+\mathrm{CoA}$ (2) $\begin{gathered}\text { 3-acetyl } \\ \text { chloramphenicol }\end{gathered} \begin{gathered}1 \text {-acetyl } \\ \text { chloramphenicol }\end{gathered}$
(3) 1 -acetyl chloramphenicol
+ acetyl CoA $\stackrel{\begin{array}{c}\text { chloramphenicol } \\ +\mathrm{CoA}\end{array}}{\longrightarrow}$ 
Previous studies with $H$. influenzae have used indirect methods of determining CAT activity, which do not identify metabolites but which employ a spectrophotometric technique to measure the chloramphenicol-dependent appearance of free coenzyme A (Roberts et al., 1980). The chromatographic approach (HPLC) enabled us to measure directly the disappearance of chloramphenicol and the appearance of each metabolite. The donor strains of $H$. influenzae and all their transconjugants produced only monoacetyl compounds and not the diacetyl compound. We believe that this is the first occasion in which the metabolites produced by CAT have been assayed directly in $H$. influenzae.

The production of 1,3-diacetyl chloramphenicol is known to be $\mathrm{pH}$ dependent, observed readily at pH 7.8 but not at pH 6.8 (Lovering et al., 1986). However our experiments were all performed at $\mathrm{pH} 7 \cdot 8$, and the addition of purified type-I CAT from $E$. coli to the reaction mixtures resulted in the subsequent production of 1,3-diacetyl chloramphenicol. Neither increasing the $\mathrm{pH}$ of the reaction mixture nor the duration of the experiment produced any detectable diacetyl metabolite in the absence of the added type-I (E. coli) CAT.

Two early studies called attention to the likelihood that some bacteria containing CAT, such as staphylococci and pseudomonads, might be unable to produce 1,3-diacetyl chloramphenicol (Suzuki et al., 1966; Okamato et al., 1967). It was suggested

\section{REFERENCES}

Breeze A S, Simpson A M 1982 An improved method using acetyl coenzyme A regeneration for the enzymic inactivation of aminoglycosides prior to sterility testing. Journal of Applied Bacteriology 53: 277-284.

Brent D A, Chandrasurin P, Ragouzeos A, Hurlbert B S, Burke J T 1980 Rearrangement of chloramphenicol-3-monosuccinate. Journal of Pharmaceutical Sciences 69 : 906-908.

Britz M L, Wilkinson R G 1978 Chloramphenicol acetyltransferase of Bacteroides fragilis. Antimicrobial Agents and Chemotherapy 14: 105-111.

Burns J L, Mendelman P M, Levy J, Stull T L, Smith A L 1985 A permeability barrier as a mechanism of chloramphenicol resistance in Haemophilus influenzae. Antimicrobial Agents and Chemotherapy 27: 46-54.

Iyobe S, Kono M, Oara K, Hashimoto H, Mitsuhashi S 1974 Relationship between chloramphenicol acetyltransferase activity and the number of resistance genes. Antimicrobial Agents and Chemotherapy 5: 68-74.

Kado C I, Liu S-T 1981 Rapid procedure for detection and isolation of large and small plasmids. Journal of Bacteriology 145: 1365-1373.

Kleanthous C, Shaw W V 1984 Analysis of the mechanism of chloramphenicol acetyltransferase by steady state kinetics. Evidence for a ternary-complex mechanism. Biochemical Journal 223: 211-220. subsequently that these experiments may have been performed under sub-optimal conditions for the production of the diacetyl metabolite (Nakagawa et al., 1979). A variant of CAT from Bacteroides fragilis was also reported to produce only the 1acetyl and 3-acetyl metabolites (Britz and Wilkinson, 1978). However the reaction may not have been left for an adequate duration because the second acetylation is known to occur at a much slower rate than that of the initial enzymic step (Shaw, 1983). It should be noted, however, that bacteria need not diacetylate chloramphenicol in order to gain resistance, because the monoacetyl metabolites are inactive as antibiotics. Viewed in such a light the second acetylation may be seen as wasteful of energy, using two moles of thioester to modify chloramphenicol rather than only the one necessary for inactivation of the antibiotic.

It is possible that those CAT variants which do not make 1,3-diacetyl chloramphenicol, including that from $H$. influenzae, may not be able to accommodate 1-acetyl chloramphenicol as a substrate because of a difference in the structural configuration of the active site (Leslie et al., 1988).

We thank Drs J. Naidoo and K. Ellis for the plasmid studies, Dr T. Howard for providing the $H$. influenzae strain W1-4 and Mr A. M. Lovering for much helpful technical advice. We are especially grateful to $\mathrm{Mr} \mathrm{E}$. Gemidjioglu for his assistance with the HPLC and to Professor W. V. Shaw for advice during preparation of the manuscript.

van Klingeren B, van Embden J D A, Dessens-Kroon M 1977 Plasmid mediated chloramphenicol resistance in Haemophilus influenzae. Antimicrobial Agents and Chemotherapy 11 : 383-387.

Leslie A G W, Moody P C E, Shaw W V 1988 The structure of chloramphenicol acetyltransferase at $1.75 \mathrm{~A}$ resolution. Proceedings of the National Academy of Sciences of the USA 85: 4133-4137.

Lovering A M, White L O, Reeves D S 1986 The assay of chloramphenicol acetyltransferase activity by high performance liquid chromatography. Journal of Antimicrobial Chemotherapy 17: 821-825.

Marqués J C, Garcia-Tornel S, Gairi J M 1984 Invasive infections caused by multiply resistant Haemophilus influenzae type b. Journal of Paediatrics 104: 162.

Nakagawa Y 1981 Studies on enzymatic acetylation of chloramphenicol by chloramphenicol-resistant bacteria: GCmass analysis. Nippon Saikingaku Zasshi 36: 747-755 (in Japanese).

Nakagawa Y, Nitahara Y, Miyamura S 1979 Kinetic studies on enzymatic acetylation of chloramphenicol in Streptococcus faecalis. Antimicrobial Agents and Chemotherapy 16: 719 723.

Okamoto S, Suzuki Y, Mise K, Nakaya R 1967 Occurrence of chloramphenicol-acetylating enzymes in various Gramnegative bacilli. Journal of Bacteriology 94 : 1616-1622.

Powell M, Koutsia-Carouzou C, Voutsinas D, Seymour A, Williams J D 1987 Resistance of clinical isolates of 
Haemophilus influenzae in United Kingdom 1986. British Medical Journal 295: 176-179.

Roberts M C, Swenson C D, Owens L M, Smith A L 1980 Characterization of chloramphenicol resistant Haemophilus influenzae. Antimicrobial Agents and Chemotherapy 18: 610 615.

Shaw W V 1967 The enzymatic acetylation of chloramphenicol by extracts of $\mathbf{R}$ factor-resistant Escherichia coli. Journal of Biological Chemistry 242: 687-693.

Shaw W V 1983 Chloramphenicol acetyltransferase: enzymology and molecular biology. CRC Critical Reviews in Biochemistry 14: 1-46.

Shaw W V, Bouanchaud D H, Goldstein F W 1978 Mechanism of transferable resistance to chloramphenicol in Haemophi- lus parainfluenzae. Antimicrobial Agents and Chemotherapy 13: 326-330.

Shaw W V, Brodsky R F 1968 Characterisation of chloramphenicol acetyltransferase from chloramphenicol-resistant Staphylococcus aureus. Journal of Bacteriology 95 : 28-36.

Stuy J H 1979 Plasmid transfer in Haemophilus influenzae. Journal of Bacteriology 139 : 520-529.

Stuy J H 1980 Chromosomally integrated conjugative plasmids are common in antibiotic-resistant Haemophilus influenzae. Journal of Bacteriology 142: 925-930.

Suzuki Y, Okamoto S, Kono M 1966 Basis of chloramphenicol resistance in naturally isolated resistant staphylococci. Journal of Bacteriology 92: 798-799. 